\title{
Structural evolution in Pt isotopes with the interacting boson model Hamiltonian derived from the Gogny energy density functional
}

\author{
K. Nomura, ${ }^{1}$ T. Otsuka,,${ }^{1,2,3}$ R. Rodríguez-Guzmán, ${ }^{4}$ L. M. Robledo, ${ }^{5}$ and P. Sarriguren ${ }^{4}$ \\ ${ }^{1}$ Department of Physics, University of Tokyo, Hongo, Bunkyo-ku, Tokyo 113-0033, Japan \\ ${ }^{2}$ Center for Nuclear Study, University of Tokyo, Hongo, Bunkyo-ku, Tokyo 113-0033, Japan \\ ${ }^{3}$ National Superconducting Cyclotron Laboratory, Michigan State University, East Lansing, Michigan 48824, USA \\ ${ }^{4}$ Instituto de Estructura de la Materia, CSIC, Serrano 123, E-28006 Madrid, Spain \\ ${ }^{5}$ Departamento de Física Teórica, Universidad Autónoma de Madrid, E-28049 Madrid, Spain \\ (Received 22 October 2010; revised manuscript received 13 December 2010; published 25 January 2011)
}

\begin{abstract}
Spectroscopic calculations are carried out for the description of the shape/phase transition in Pt nuclei in terms of the interacting boson model (IBM) Hamiltonian derived from (constrained) Hartree-Fock-Bogoliubov (HFB) calculations with the finite range and density-dependent Gogny-D1S energy density functional. Assuming that the many-nucleon driven dynamics of nuclear surface deformation can be simulated by effective bosonic degrees of freedom, the Gogny-D1S potential energy surface (PES) with quadrupole degrees of freedom is mapped onto the corresponding PES of the IBM. By using this mapping procedure, the parameters of the IBM Hamiltonian, relevant to the low-lying quadrupole collective states, are derived as functions of the number of valence nucleons. Merits of both Gogny-HFB and IBM approaches are utilized so that the spectra and the wave functions in the laboratory system are calculated precisely. The experimental low-lying spectra of both ground-state and sideband levels are well reproduced. From the systematics of the calculated spectra and the reduced $E 2$ transition probabilities $B(E 2)$, the prolate-to-oblate shape/phase transition is shown to take place quite smoothly as a function of neutron number $N$ in the considered Pt isotopic chain, for which the $\gamma$ softness plays an essential role. All of these spectroscopic observables behave consistently with the relevant PES and the derived parameters of the IBM Hamiltonian as functions of $N$. Spectroscopic predictions are also made for those nuclei that do not have enough experimental $E 2$ data.
\end{abstract}

DOI: 10.1103/PhysRevC.83.014309

PACS number(s): 21.10.Re, 21.60.Ev, 21.60.Fw, 21.60.Jz

\section{INTRODUCTION}

The quadrupole collective motion, which is essentially the multifermion dynamics of the surface deformation, has always attracted considerable attention in nuclear physics [1-3]. The nuclear surface can change according to the number of valence nucleons and exhibits distinct regularities, often recognized as a signature of shape/phase transition [4]. Nevertheless, a fully microscopic understanding of the evolution of the nuclear shapes with the number of nucleons still remains a major challenge [5-14]. From the experimental point of view, low-lying spectroscopy is one of the most powerful sources of information about structural evolution and/or shape transitions in atomic nuclei because it allows us to establish signatures correlating the excitation energies with deformation properties [15-21]. In particular, the complex interplay between several deformation degrees of freedom, taking place in different regions of the nuclear chart, offers the possibility of testing microscopic descriptions of atomic nuclei under a wide variety of conditions. In this context, mean-field approximations based on effective energy density functionals (EDFs), which are a cornerstone to almost all microscopic approximations to the nuclear many-body problem [3], appear to be a first tool to rely on when looking for fingerprints of nuclear shape/phase transitions.

Mean-field approximations are based on product trial wave functions, which are used to minimize a given EDF. Such products break several symmetries of the underlying nuclear Hamiltonian (spontaneous symmetry-breaking mechanism), allowing the use of an enlarged Hilbert space within which static correlations associated with collective modes (e.g., quadrupole deformations) are incorporated at the cost of a moderate effort. Nowadays, systematic mean-field studies are possible because, on one hand, important advances have been made in the fitting protocols providing EDFs with global predictive power all over the nuclear chart. Popular EDFs for calculations along these lines are the nonrelativistic Gogny [22,23] and Skyrme [7,24,25] EDFs, as well as different parametrizations of the relativistic mean-field Lagrangian $[7,26]$. On the other hand, it has also become possible to recast mean-field equations in terms of efficient minimization procedures such as the so-called gradient method $[27,28]$. One of the advantages of the gradient method is the way that it handles constraints, which is well adapted to the case where a large number of constraints are required (such as the case that requires, in addition to the protonand neutron-number constraints, constraints on both $\beta$ and $\gamma$ degrees of freedom characterizing the nuclear shape). Another advantage is its robustness in reaching a solution, a convenient property when large-scale calculations requiring the solution of many HFB equations are performed.

On its own, the interacting boson model (IBM) [29] has been quite successful in reproducing the experimental spectra and electromagnetic transitions for low-lying quadrupole collective states. The virtue of the IBM is, in its simplicity, its robust capability of calculating the spectroscopic observables precisely, while the parameters of the IBM Hamiltonian 
have been determined phenomenologically. Therefore, the IBM itself has a certain microscopic foundation, where the collective $J=0^{+}(S)$ and $2^{+}(D)$ pairs of valence nucleons are approximated by $J=0^{+}(s)$ and $2^{+}(d)$ bosons, respectively [30]. The proton and neutron degrees of freedom can be taken into account, where the so-called proton (neutron) $s_{\pi}$ and $d_{\pi}\left(s_{\nu}\right.$ and $\left.d_{\nu}\right)$ bosons correspond to the collective pairs of valence protons (neutrons) $S_{\pi}$ and $D_{\pi}\left(S_{v}\right.$ and $\left.D_{v}\right)$ [30,31]. This is closer to a microscopic picture compared to a simpler version of IBM and is known as the proton-neutron interacting boson model (IBM-2). As the number of valence protons (neutrons) is constant for a given nucleus, the number of proton (neutron) bosons, denoted by $n_{\pi}\left(n_{\nu}\right)$, is set equal to half of the valence proton (neutron) numbers. The derivation of the IBM Hamiltonian has been studied extensively in realistic cases for nearly spherical or $\gamma$-unstable shapes [32-35] using generalized seniority states of the shell model [30,31], as well as for deformed nuclei $[36,37]$, but it still remains to be done for general cases in a unified manner. Therefore, it is timely and necessary to bridge the IBM and mean-field models with the help of fermion-to-boson mapping procedures. The key question here is to investigate to which extent the underlying fermionic dynamics of mean-field models can be translated into effective bosonic degrees of freedom. Such an approach would enable one to take advantage of the universality of microscopic nuclear EDFs [7,22-26] and the simplicity of the IBM [29]. By the combination of both models, one would be able to access the spectroscopic observables that have the good quantum numbers in the laboratory system, including those for experimentally unexplored nuclei.

A different way of deriving the parameters of the IBM Hamiltonian has been recently proposed by two of us [38]. The IBM Hamiltonian has been constructed by mapping the mean-field potential energy surface (PES), obtained in the framework of (constrained) Skyrme Hartree-Fock plus BCS calculations [39], onto the corresponding PES of the IBM. The parameters of the IBM Hamiltonian, relevant to the description of the considered quadrupole collective states, have been shown to be determined uniquely as a function of the number of valence nucleons by using the wavelet analysis [40]. Calculations along these lines have been performed to study the shape/phase transition in Sm isotopes with neutron number $N=82 \sim 96$, as well as for $\mathrm{Ba}, \mathrm{Xe}, \mathrm{Ru}$, and $\mathrm{Pd}$ isotopes with $N=50 \sim 82$. Spectroscopic predictions have also been made for W and Os nuclei with $N>126$ in the lower-right quadrant of ${ }^{208} \mathrm{~Pb}[38,40]$. In addition, it has to be mentioned that the quantum-mechanical correlation effect on the binding energies can be included in such calculations by diagonalizing the mapped IBM Hamiltonian [40]. Note that, in this framework, the IBM keeps its important properties, including the boson number counting rule as well as the algebraic features.

In this paper, we present spectroscopic calculations for the $\mathrm{Pt}$ isotopic chain (i.e., for the even-even isotopes ${ }^{172-200} \mathrm{Pt}$ ) in terms of an IBM Hamiltonian determined microscopically by mapping the PES obtained in the framework of the (constrained) Hartree-Fock-Bogoliubov (HFB) approximation $[10,12,28]$ based on the parametrization D1S [41] of the Gogny-EDF [22,23]. Quite recently, the structural evolution in $\mathrm{Pt}$ isotopes, including the role of triaxiality (i.e., the $\gamma$ degree of freedom), has been studied by three of us [42]. In addition to the (standard) Gogny-D1S EDF, the new incarnations D1N [43] and D1M [44] of the Gogny-EDF have also been included in the mean-field analysis of Ref. [42]. The considered range of neutron numbers included prolate, triaxial, oblate, and spherical shapes and served as a detailed comparison of the (mean-field) predictions of the new parameter sets D1N and D1M against the standard parametrization D1S. It has been shown that, regardless of the particular version of the Gogny-EDF employed, the prolate-to-oblate shape/phase transition occurs quite smoothly with the $\gamma$ softness playing an important role. It is, therefore, very interesting to study how the systematics of the HFB PES discussed in Ref. [42] is reflected in the isotopic evolution of the corresponding low-lying quadrupole collective states, and how accurately such states can be reproduced by a mapped IBM Hamiltonian $[38,40]$. Let us stress that our main goal in this work is to study the performance of a fermion-to-boson mapping procedure $[38,40]$ based on the Gogny-EDF. For this reason, as a first step, we will restrict ourselves to a mapping in terms of the parametrization Gogny-D1S already considered as global and able to describe reasonably well low-energy experimental data all over the nuclear chart (see, for example, Refs. [28,45] and references therein).

From the theoretical perspective, the $\mathrm{Pt}$ and neighboring isotopic chains have been extensively studied in terms of both IBM and mean-field-based approaches. There is much experimental evidence $[46,47]$ revealing existences of $\gamma$ unstable $\mathrm{O}(6)$ nuclei in Pt isotopes. The IBM-2 has been used in a phenomenological way for the spectroscopy of $\mathrm{Pt}$, Os, and $\mathrm{W}$ isotopes [48,49]. The prolate-to-oblate transition in $\mathrm{Pt}$, as well as in Os and $\mathrm{W}$ nuclei, has been observed in a recent experiment [50], where a relatively moderate oblate-to-prolate shape/phase transition occurs in $\mathrm{Pt}$ as compared to Os and $\mathrm{W}$ nuclei. Spectroscopic calculations have been carried out for $\mathrm{Pt}$ isotopes in the framework of the five-dimensional collective Hamiltonian, derived from the pairing-plus-quadrupole model [51]. Evidence for $\gamma$ vibrations and shape evolution in ${ }^{184-190} \mathrm{Hg}$ has been considered in Ref. [52], where a five-dimensional collective Hamiltonian was built with the help of constrained Gogny-D1S HFB calculations. On the other hand, systematic mean-field studies of the evolution of the ground-state shapes in $\mathrm{Pt}$ and the neighboring $\mathrm{Yb}$, $\mathrm{Hf}, \mathrm{W}$, and Os nuclei have been carried out with nonrelativistic Skyrme [10] and Gogny [28,42] EDFs, as well as within the framework of the relativistic mean-field (RMF) approximation [53]. One should also keep in mind that Pt, $\mathrm{Pb}$, and $\mathrm{Hg}$ nuclei belong to a region of the nuclear chart, around the proton shell closure $Z=82$, characterized by a pronounced competition between low-lying configurations corresponding to different intrinsic deformations [54]; therefore, a detailed description of the very rich structural evolution in these nuclei requires the inclusion of correlations beyond the static mean-field picture [55-57], accounting for both symmetry restoration and configuration mixing. The role of configuration mixing in this region has also been considered in phenomenological IBM studies [58-60].

This paper is organized as follows. In Sec. II, we will briefly describe the theoretical tools used in this 
study. Illustrative examples of the IBM PES, obtained by mapping the corresponding Gogny-HFB PES, are presented in Sec. III. The isotopic evolution of the IBM parameters derived for the nuclei ${ }^{172-200} \mathrm{Pt}$ is discussed in Sec. IV. Spectroscopic calculations, including the systematics of excitation spectra and reduced $E 2$ transition probabilities $B(E 2)$ along the $\mathrm{Pt}$ isotopic chain, will be discussed in Sec. V. There, we will also show detailed comparisons between the predicted level schemes and the available data for some Pt isotopes selected as a representative sample. Finally, Sec. VI is devoted to the concluding remarks and work perspectives.

\section{THEORETICAL PROCEDURE}

In this section, we briefly describe the theoretical frameworks used in this study, i.e., the constrained HFB approximation, as well as the procedure followed to construct the corresponding mapped IBM Hamiltonian. For more details, the reader is referred to Refs. [28,42] and [40].

In order to compute the Gogny-HFB PESs, which are our starting point, we have used the (constrained) HFB method together with the parametrization D1S of the Gogny-EDF. The solution of the HFB equations, leading to the set of vacua $\left|\Phi_{\mathrm{HFB}}(\beta, \gamma)\right\rangle$, is based on the equivalence of the HFB with a minimization problem that is solved using the gradient method [27,28]. In agreement with the fitting protocol of the force, the kinetic energy of the center-of-mass motion has been subtracted from the Routhian to be minimized in order to ensure that the center of mass is kept at rest. The exchange Coulomb energy is considered in the Slater approximation, and we neglect the contribution of the Coulomb interaction to the pairing field. The HFB quasiparticle operators are expanded in a harmonic-oscillator ( $\mathrm{HO}$ ) basis containing a sufficient number of shells (i.e., $N_{\text {shell }}=13$ major shells) to grant convergence for all values of the mass quadrupole operators and for all the nuclei studied. We constrain the average values of the mass quadrupole operators $\hat{Q}_{20}=\frac{1}{2}\left(2 z^{2}-x^{2}-y^{2}\right)$ and $\hat{Q}_{22}=\frac{\sqrt{3}}{2}\left(x^{2}-y^{2}\right)$ to the desired deformation values $Q_{20}$ and $Q_{22}$ defined as

$$
Q_{20}=\left\langle\Phi_{\mathrm{HFB}}\left|\hat{Q}_{20}\right| \Phi_{\mathrm{HFB}}\right\rangle
$$

and

$$
Q_{22}=\left\langle\Phi_{\mathrm{HFB}}\left|\hat{Q}_{22}\right| \Phi_{\mathrm{HFB}}\right\rangle .
$$

In Ref. [42], the $Q-\gamma$ energy contour plots

$$
Q=\sqrt{Q_{20}^{2}+Q_{22}^{2}}
$$

and

$$
\tan \gamma=\frac{Q_{22}}{Q_{20}}
$$

have been used to study the (mean-field) evolution of the ground-state shapes in Pt nuclei. Alternatively, one could also consider the $\beta-\gamma$ representation in which the quadrupole deformation parameter $\beta$ is written [28] in terms of $Q$ [Eq.(3)] as

$$
\beta=\sqrt{\frac{4 \pi}{5}} \frac{Q}{A\left\langle r^{2}\right\rangle},
$$

where $\left\langle r^{2}\right\rangle$ represents the mean-squared radius evaluated with the corresponding HFB state $\left|\Phi_{\mathrm{HFB}}\right\rangle$.

The set of constrained HFB calculations described provides the Gogny-D1S $\beta-\gamma$ PES [i.e., the total HFB energies $\left.E_{\mathrm{HFB}}(\beta, \gamma)[3]\right]$ required for the subsequent mapping procedure, for which the following IBM-2 Hamiltonian $\hat{H}_{\mathrm{IBM}}$ is employed:

$$
\hat{H}_{\mathrm{IBM}}=\epsilon\left(\hat{n}_{d \pi}+\hat{n}_{d v}\right)+\kappa \hat{Q}_{\pi} \cdot \hat{Q}_{\nu},
$$

where

$$
\hat{n}_{d \rho}=d_{\rho}^{\dagger} \cdot \tilde{d}_{\rho} \quad(\rho=\pi, v)
$$

and

$$
\hat{Q}_{\rho}=\left[s_{\rho}^{\dagger} \tilde{d}_{\rho}+d_{\rho}^{\dagger} \tilde{s}_{\rho}\right]^{(2)}+\chi_{\rho}\left[d_{\rho}^{\dagger} \tilde{d}_{\rho}\right]^{(2)}
$$

stand for the $d$-boson number operator and the quadrupole operator, respectively. The competition between the coupling constants $\epsilon$ and $\kappa$ determines the degree of nuclear deformation.

The bosonic PES is represented by the expectation value of $\hat{H}_{\mathrm{IBM}}$, computed in terms of the so-called boson coherent state [61-63]

$$
|\Phi\rangle \propto \prod_{\rho=\pi, \nu}\left(s_{\rho}^{\dagger}+\sum_{\mu=0, \pm 2} \alpha_{\rho \mu} d_{\rho \mu}^{\dagger}\right)^{n_{\rho}}|0\rangle,
$$

where $|0\rangle$ stands for the boson vacuum (i.e., inert core) and the coefficients $\alpha$ are expressed as $\alpha_{\rho 0}=\beta_{\rho} \cos \gamma_{\rho}, \alpha_{\rho \pm 1}=0$, and $\alpha_{\rho \pm 2}=\frac{1}{\sqrt{2}} \beta_{\rho} \sin \gamma_{\rho}$. Within this context, the intrinsic shape of the nucleus is described in terms of the (axially symmetric) deformation $\beta_{\rho}$ and the (triaxial) deformation $\gamma_{\rho}$. In this study, as well as in our previous works [38,40], we assume for simplicity that $\beta_{\pi}=\beta_{\nu} \equiv \beta_{B}$ and $\gamma_{\pi}=\gamma_{\nu} \equiv \gamma_{\mathrm{B}}$. The IBM $\mathrm{PES}$ is then given by $[38,40]$

$$
\begin{aligned}
& E_{\mathrm{IBM}}\left(\beta_{B}, \gamma_{B}\right) \\
& =\frac{\epsilon\left(n_{\pi}+n_{\nu}\right) \beta_{B}^{2}}{1+\beta_{B}^{2}}+n_{\pi} n_{\nu} \kappa \frac{\beta_{B}^{2}}{\left(1+\beta_{B}^{2}\right)^{2}} \\
& \quad \times\left[4-2 \sqrt{\frac{2}{7}}\left(\chi_{\pi}+\chi_{\nu}\right) \beta_{B} \cos 3 \gamma_{B}+\frac{2}{7} \chi_{\pi} \chi_{\nu} \beta_{B}^{2}\right] .
\end{aligned}
$$

Here, we assume the proportionality $\beta_{B}=C_{\beta} \beta$, with $C_{\beta}$ being a numerical coefficient [38]. If one further assumes the separability of the mapping along the $\beta$ and $\gamma$ directions $[38,40]$, one then has $\gamma_{B}=\gamma$. Thus, $\left(\beta_{B}, \gamma_{B}\right)$ represent the boson images of the (fermion) deformation parameters $(\beta, \gamma)$ given by Eqs. (4) and (5). We then map a point $(\beta, \gamma)$ on the HFB PES, within an energy range relevant for the considered low-lying quadrupole collective states, onto the corresponding point $\left(\beta_{B}, \gamma_{B}\right)$ on the IBM PES. This process is exactly the mapping of the fermionic PES onto the bosonic PES. In practice, one determines the $\epsilon, \kappa, \chi_{\pi, \nu}$, and $C_{\beta}$ values for each individual nucleus by drawing the IBM PES so that the 
topology of the corresponding HFB PES is reproduced. This is done unambiguously by means of the recently developed procedure [40], which makes use of the powerful method of the wavelet transform [64].

Here we make the following remarks: The topology of the HFB PES reflects essential (fermionic) features of manynucleon systems, such as the Pauli principle and the underlying nuclear interactions. Such effects are supposed to be incorporated into the boson system by the mapping procedure [38]. On the other hand, the solution of the five-dimensional (5D) collective Bohr Hamiltonian with parameters obtained from EDF calculations (see, for example, $[45,65,66]$ ) is a popular alternative to obtain the low-lying collective spectra in eveneven nuclei. In this kind of calculation, the pure mean-field PES is replaced by another quantity that incorporates, in addition to the HFB energy, the zero-point rotational and vibrational corrections. These corrections to the energy are intimately related to the use of a generalized kinetic energy term for the collective motion, which includes not only moments of inertia but also collective vibrational masses. To what extent the present mapping procedure plus the solution of the IBM Hamiltonian is able to mimic the solution of the 5D Bohr Hamiltonian is still an open question, which can be partially answered by looking at the reasonable results obtained with our method and compare qualitatively well with those of the 5D Hamiltonian. A possible way to incorporate the effect of the collective masses into the mapping would be to make a change of variables (analogous to the one invoked in the derivation of the gaussian overlap approximation (GOA) [3]) as to render the collective masses constant all over the range of allowed values of the $\beta$ and $\gamma$ deformation parameters. Perhaps, this is the missing element that could correct some observed $[38,40]$ systematic deviations of the IBM rotational spectra with respect to the experimental ones for well-deformed systems, and requires the introduction of an additional mass term in the IBM Hamiltonian known as the $L \cdot L$ term [67]. However, this problem does not show up for moderately deformed cases such as those studied in this work and, therefore, it is not considered here.

\section{POTENTIAL ENERGY SURFACES}

The IBM PESs obtained for the nuclei ${ }^{180-198} \mathrm{Pt}$ are shown in Fig. 1 as a representative sample. The Gogny-D1S PESs, also shown as references in Fig. 1, are identical to those presented in Fig. 2 of Ref. [42]. The IBM parameters $\epsilon, \kappa$, $\chi_{\pi, v}$, and $C_{\beta}$, to be discussed later on in Sec. IV, have been obtained by mapping the corresponding Gogny-D1S PESs, obtained in the framework of HFB calculations along the lines previously described in Sec. II.

The IBM PESs from ${ }^{180} \mathrm{Pt}$ to ${ }^{186} \mathrm{Pt}$ display a prolatedeformed minimum and an oblate-deformed saddle point. The prolate minimum becomes softer in the $\gamma$ but steeper in the $\beta$ direction as the number of neutrons increases. This is, roughly speaking, consistent with the topologies of the HFB PESs, where the minima are located a bit off but quite nearby the line $\gamma=0^{\circ}$.

The IBM PESs for both ${ }^{188,190} \mathrm{Pt}$ are $\gamma$ soft, having the minimum on the oblate side. These nuclei are supposed to be close to the critical point of the prolate-to-oblate shape transition. The corresponding HFB PESs display shallow triaxial minima with $\gamma \sim 30^{\circ}$ and are also soft along the $\gamma$ direction. The IBM Hamiltonian considered in this study does not provide a triaxial minimum but, instead, provides either a prolate or oblate minimum, as can be seen from Eq. (10). The $\gamma$ softness can be simulated by choosing the parameters $\chi_{\pi}$ and $\chi_{v}$ so that their sum becomes nearly equal to zero. This is reasonable when a triaxial minimum is not deep enough as in the present case, where the triaxial minimum point in the HFB PES differs by at most several hundred kiloelectronvolts in energy from either a prolate or oblate saddle point. However, the topology of the mapped IBM PES is then somewhat sensitive to the values of the parameters $\chi_{\pi}$ and $\chi_{\nu}$, which occasionally results in a quantitative difference in the location of the minimum in the IBM PES from that of the HFB PES. In fact, and contrary to what happens with the HFB PES, the IBM PES of ${ }^{190} \mathrm{Pt}$ is softer in $\gamma$ than that of ${ }^{188} \mathrm{Pt}$. One should then expect a certain deviation of the resultant IBM spectra from the experimental spectra, which can be partly attributed to the small difference already mentioned.

In Fig. 1, isotopes from ${ }^{192} \mathrm{Pt}$ to ${ }^{198} \mathrm{Pt}$ exhibit oblate deformation. The locations of their energy minima and their curvatures in both $\beta$ and $\gamma$ directions agree well with those of the Gogny-D1S PESs. These isotopes become steeper in the $\gamma$ direction and shallower in the $\beta$ direction as the number of neutrons increases. Their energy minima approach the origin more rapidly than the lighter Pt nuclei shown Fig. 1. This evolution reflects the transition from oblate-deformed ground states to a spherical vibrator as one approaches the neutron shell closure $N=126$.

\section{DERIVED IBM PARAMETERS}

The IBM parameters $\epsilon, \kappa, \chi_{\pi, v}$, and $C_{\beta}$ derived for the nuclei ${ }^{172-200} \mathrm{Pt}$ from the mapping procedure described in Sec. II are depicted in Figs. 2(a)-2(d) as functions of the mass number $A$.

Figure 2(a) shows that the parameter $\epsilon$ gradually decreases toward mid-shell in accordance with the growth of the deformation. This trend reflects the structural evolution from nearly spherical to more deformed shapes, and is consistent with previous results for other isotopic chains [40]. In Fig. 2(b), the derived $\kappa$ parameter is almost constant and somewhat larger in comparison with the phenomenological value [49], which is the consequence of the sharp potential valleys observed in the Gogny-D1S PESs.

On the other hand, in Fig. 2(c), the proton parameter $\chi_{\pi}$ is almost constant while the neutron parameter $\chi_{v}$ changes significantly. The systematic behavior of the present $\chi_{v}$ value is consistent with the phenomenological behavior [49], while there is quantitative difference between the former and the latter. The magnitude of the sum $\chi_{\pi}+\chi_{\nu}$, as well as its sign, depend on how sharp the HFB PES is in the $\gamma$ direction and on whether the nucleus is prolate (negative sum) or oblate (positive sum) deformed, respectively. Therefore, as $\chi_{\pi}$ does not change much, the role of $\gamma$ instability can be seen clearly from the systematics of $\chi_{v}$. For the isotopes ${ }^{172-180} \mathrm{Pt}$, the 


\section{Potental Energy Surfaces (HFB)}

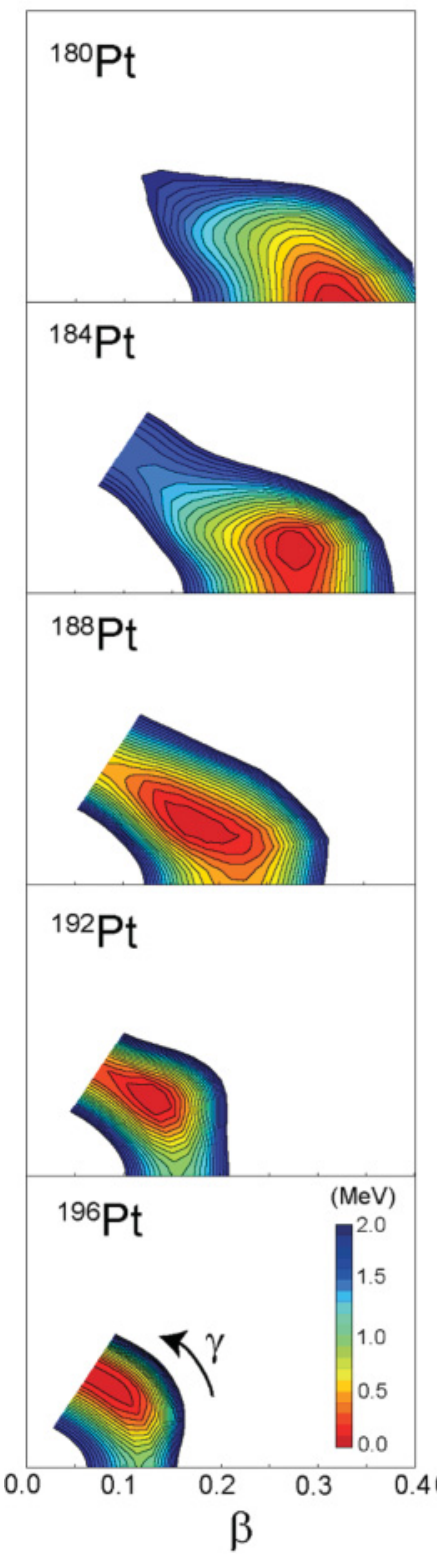

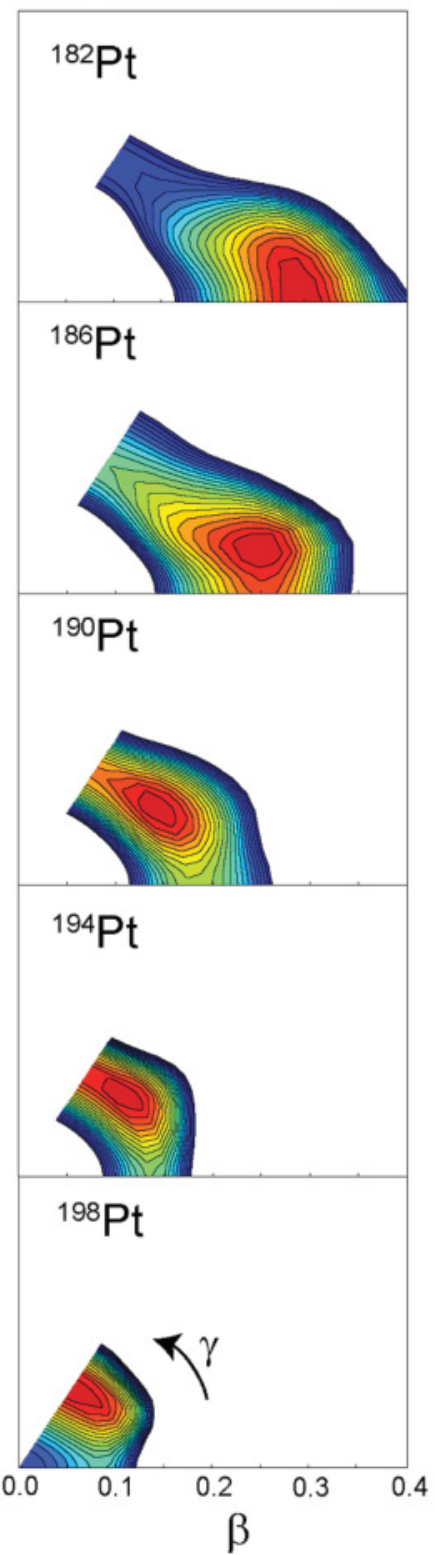

Potental Energy Surfaces (IBM)
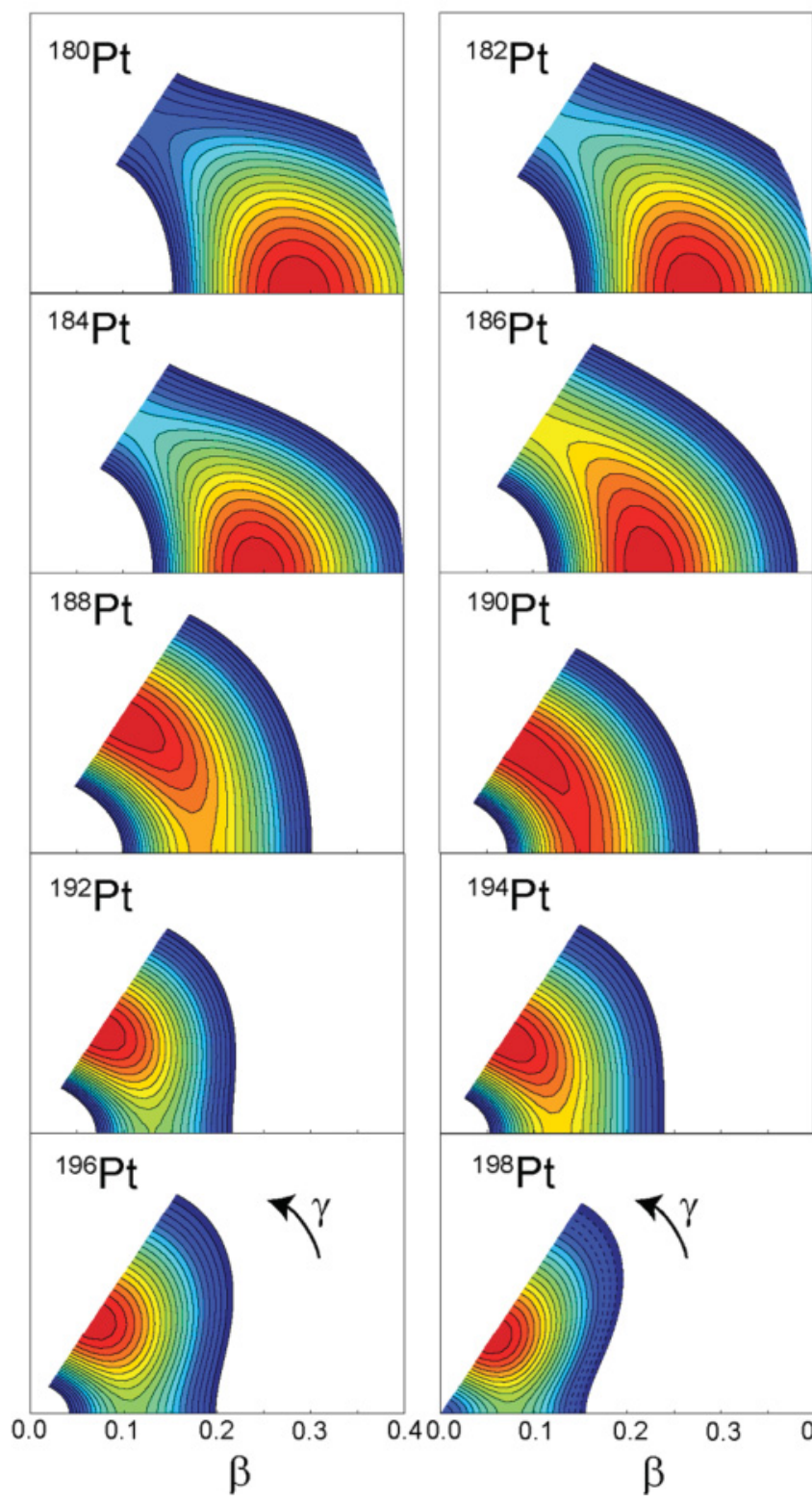

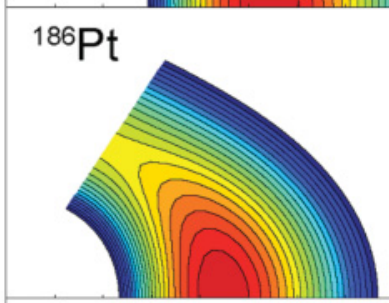

${ }^{190} \mathrm{Pt}$
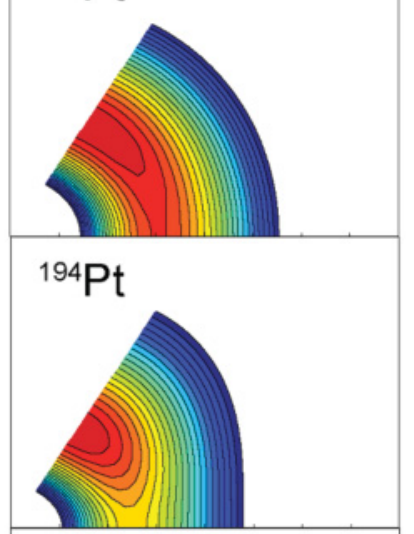

${ }^{198} \mathrm{Pt}$

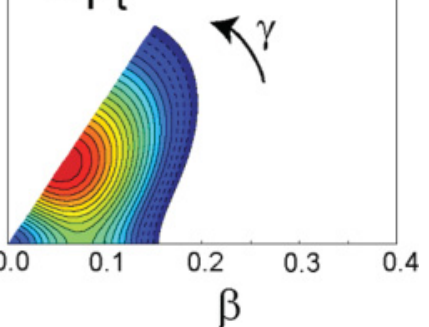

FIG. 1. (Color online) HFB and IBM PESs in the $\beta \gamma$ plane for the nuclei ${ }^{180-198} \mathrm{Pt}$. In the latter case, $\gamma=\gamma_{B}$ and $\beta=\beta_{B} / C_{\beta}$. The PESs are shown within $0.00 \leqslant \beta \leqslant 0.40$ and $0^{\circ} \leqslant \gamma \leqslant 60^{\circ}$ up to $2 \mathrm{MeV}$ excitation from the minimum. Contour spacing is $100 \mathrm{keV}$. For details, see the main text.

PES exhibits prolate deformation and the sum $\chi_{\pi}+\chi_{\nu}$ has a negative sign. The average of the derived $\chi_{\pi}$ and $\chi_{v}$ values is nearly equal to zero for the nuclei ${ }^{182-194} \mathrm{Pt}$. This is a consequence of the $\gamma$ softness in the corresponding HFB PESs. On the other hand, the sum $\chi_{\pi}+\chi_{\nu}$ becomes larger with a positive sign as we approach the neutron shell closure $N=126$, reflecting the appearance of weakly deformed oblate structures in the corresponding PESs.

Figure 2(d) shows that $C_{\beta}$ decreases gradually toward the middle of the major shell. $C_{\beta}$ can be interpreted as the "bridge" between the geometrical deformation $\beta$ [1] and the IBM deformation $\beta_{B}$ and is thus proportional to the ratio between the total and valence nucleon numbers, in a good approximation [62]. This is probably the reason for the decreasing trend observed in Fig. 2(d), as well as in earlier studies for other isotopic chains $[38,40]$.

\section{SPECTROSCOPIC CALCULATIONS}

With all the parameters $\epsilon, \kappa, \chi_{\pi, v}$, and $C_{\beta}$ required by the IBM Hamiltonian at hand, we are now able to test the spectroscopic quality of our mapping procedure, based on the Gogny-D1S EDF, for the nuclei ${ }^{172-200} \mathrm{Pt}$. Therefore, in the following we will discuss our predictions concerning the properties of the low-lying spectra as well as the reduced 


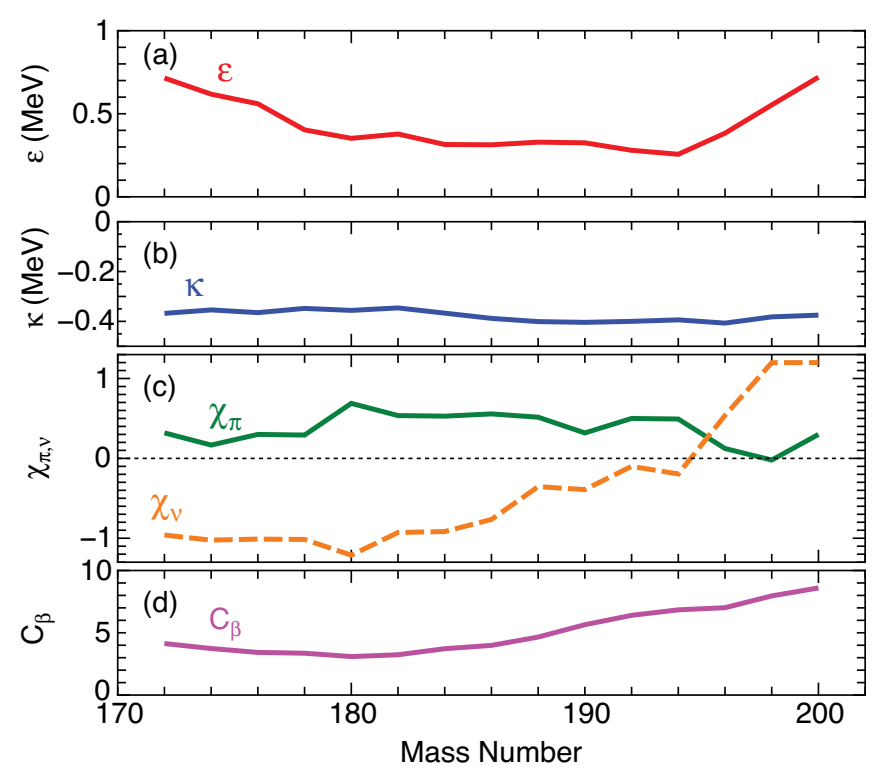

FIG. 2. (Color online) IBM parameters $\epsilon, \kappa, \chi_{\pi, v}$, and $C_{\beta}$ as functions of the mass number $A$. For the wavelet analysis, the Morlet function is used [40].

transition probabilities $B(E 2)$. We will also consider their correspondence with the mapped PESs and the derived IBM parameters. We will compare our theoretical predictions with the available experimental data taken from Brookhaven National Nuclear Data Center (NNDC) [68] and from the latest Nuclear Data Sheets [69]. The diagonalization of the IBM Hamiltonian is performed numerically for each nucleus using the code NPBOS [70].

Here, we have to note that the experimental $2_{3}^{+}$and $4_{3}^{+}$levels for mass numbers $192 \leqslant A \leqslant 200$ belong to bands different from those of the $0_{2}^{+}$level, while they are assigned to the quasi- $\beta$-band levels in Fig. 3(b), as well as in Fig. 5, for the sake of convenience. Similarly, as one will see in Fig. 3(c), the experimental data for the $3_{1}^{+}$and the $4_{2}^{+}$levels in ${ }^{198,200} \mathrm{Pt}$ are assigned to the quasi- $\gamma$-band levels lying on top of the $2_{2}^{+}$ energy.

\section{A. Evolution of low-lying spectra}

Figure 3 displays the calculated spectra for (a) groundstate, (b) quasi- $\beta$, and (c) quasi- $\gamma$ bands. What is striking is the good agreement between the present calculations and the experimental data, not only for the ground state but also for quasi- $\beta$ and quasi $-\gamma$ band energies, where overall experimental trends are reproduced fairly well, in particular for the openshell nuclei ${ }^{180-192} \mathrm{Pt}$.

We show in Fig. 3(a) the evolution of the $2_{1}^{+}, 4_{1}^{+}, 6_{1}^{+}$, and $8_{1}^{+}$levels in the considered Pt nuclei as functions of the mass number $A$. The calculated energies decrease toward the middle of the major shell with the number of the valence neutrons and remain almost constant for $176 \lesssim A \lesssim 186$ nuclei. Although these tendencies are well reproduced, the rotational features are somewhat enhanced in the calculated levels for ${ }^{180,182,184} \mathrm{Pt}$, which are slightly lower in energy than the experimental levels. From both the theoretical results and the experimental data, one can also observe clear fingerprints for structural evolution with a jump between ${ }^{186} \mathrm{Pt}$ and ${ }^{188} \mathrm{Pt}$, which can be correlated with the change of the mapped PESs from prolate to oblate deformations. For $A \geqslant 188$, the yrast levels gradually go up as the neutron shell closure $N=126$ is approached.

One can also find signatures for a shape/phase transition in the systematics of the quasi- $\beta$-band levels shown in Fig. 3(b). From $A=180$ to 186 , the $0_{2}^{+}$band head and the $2_{3}^{+}$ level look either constant or nearly constant in both theory and experiment. The two levels are pushed up rather significantly from $A=186$ to 188 consistently with the systematics in the ground-state band and with the change of the mapped PESs as functions of the neutron number $N$. The calculated $0_{2}^{+}$and $2_{3}^{+}$ levels are higher than, but still follow, the experimental trends.

Coming now to the quasi- $\gamma$-band levels shown in Fig. 3(c), one can observe the remarkable agreement between theoretical and experimental spectra for $180 \leqslant A \leqslant 186$, where the $3_{1}^{+}$
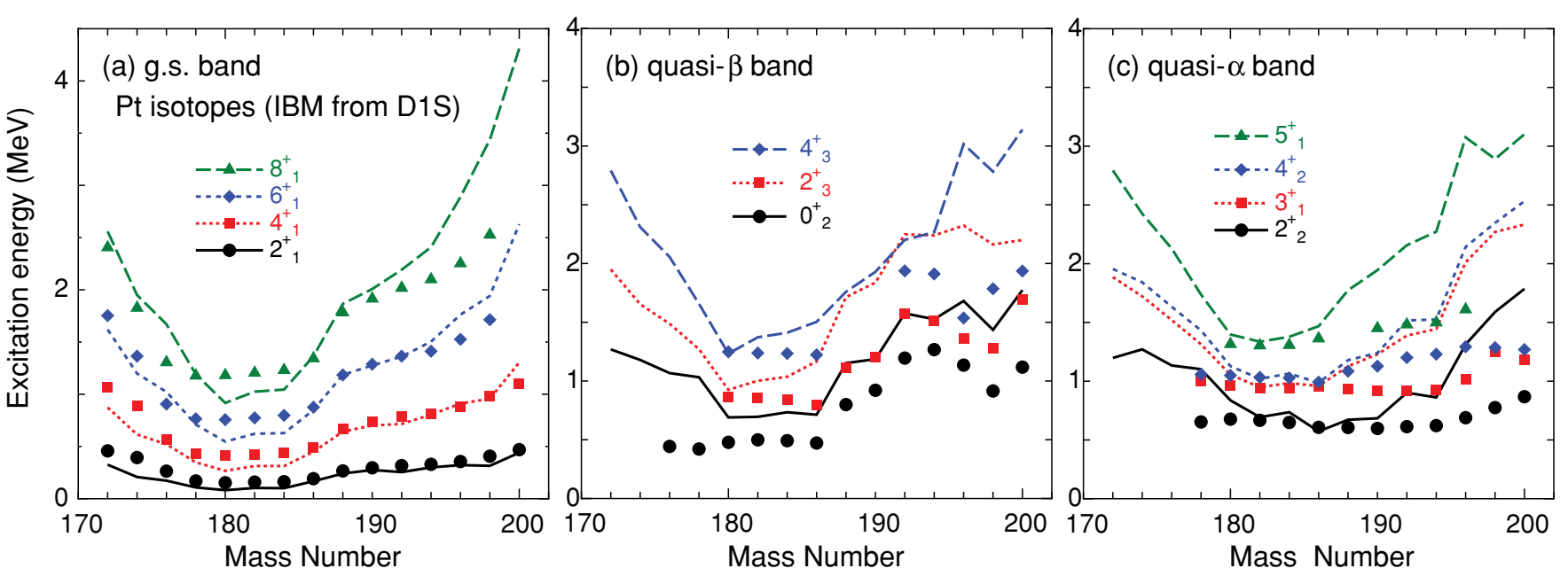

FIG. 3. (Color online) Evolution of calculated (curves) and experimental (symbols) low-lying spectra of ${ }^{172-200} \mathrm{Pt}$ nuclei for (a) ground-state, (b) quasi- $\beta$, and (c) quasi- $\gamma$ bands as functions of the mass number $A$. Experimental data are taken from Ref. [68]. 
level lies close to the $4_{2}^{+}$level. However, this calculation suggests that this trend persists even for $188 \leqslant A \leqslant 196$, whereas the relative spacing between the experimental $3_{1}^{+}$and $4_{2}^{+}$levels for these nuclei is larger. Similar deviation occurs for $5_{1}^{+}$and $6_{2}^{+}$levels, although the latter is not exhibited in Fig. 3(b). This means that our calculations suggest the feature characteristic of the $\mathrm{O}(6)$ symmetry, where the staggering occurs as $2_{\gamma}^{+},\left(3_{\gamma}^{+} 4_{\gamma}^{+}\right),\left(5_{\gamma}^{+} 6_{\gamma}^{+}\right), \ldots$, etc. However, the experimental levels are lying more regularly, particularly for $188 \leqslant A \leqslant 196$, and thus appear to be in between the $\mathrm{O}(6)$ limit and a rigid triaxial rotor where the staggering shows up as $\left(2_{\gamma}^{+} 3_{\gamma}^{+}\right),\left(4_{\gamma}^{+} 5_{\gamma}^{+}\right), \ldots$, etc. [71]. Such a deviation of the $\gamma$-band structure seems to be nothing more than a consequence of an algebraic nature of the IBM, and indeed has also been found in existing phenomenological IBM calculations [49]. From a phenomenological point of view, the so-called cubic (or the three-boson) interaction [72,73] has been useful for reproducing the experimental $\gamma$-band structure. The cubic term produces a shallow triaxial minimum that is seen in the Gogny-HFB PES, and may be introduced also in the Hamiltonian in Eq. (6). This is, however, out of focus in the current theoretical framework, because the cubic term represents an effective force, the origin of which remains to be investigated further.

Here, the deviations observed in the sideband levels (even in some of the ground-state-band levels) for $A \geqslant 196$ are probably related to the larger magnitude of the parameter $\kappa$ as compared with its phenomenological value [49]. Roughly speaking, when the magnitude of $\kappa$ becomes larger, the moment of inertia decreases, resulting in the deviation of not only the ground-state band but also the sideband energies. The problem arises in the present case partly because, in the vicinity of the shell closure $N=126$, the HFB PESs exhibit weak oblate deformations close to the origin $\beta=0$, as we showed in Fig. 1. In addition, the curvatures along the $\beta$ direction around the minima are somewhat larger. These peculiar topologies of the Gogny-D1S PES make it rather difficult to determine a value of $\kappa$ that gives reasonable agreement of sideband energies with the experimental ones. In this case, one may interpret that the deviation is mainly due to the properties of the particular version of the Gogny-EDF considered in this paper. Another possibility is that the boson Hamiltonian used may still be simple, requiring the introduction of additional interaction terms in the boson system. Investigation along these lines is in progress and will be reported elsewhere.

We note that some lighter $\mathrm{Pt}$ nuclei, as well as the neighboring $\mathrm{Hg}$ and $\mathrm{Pb}$ isotopes, are often revealed to exhibit coexistence of prolate and oblate shapes. Some existing IBM studies consider a configuration mixing, i.e., particle-hole excitation across the proton $Z=82$ shell, leading to an enlarged model space consisting of a so-called regular/single configuration (with $N_{B}$ bosons) and an intruder, deformed $2 p-2 h$ excitation configuration (with $N_{B}+2$ bosons) [58]. Along these lines, there have been debates over which is adequate for the description of low-lying structure of lighter $\mathrm{Pt}$ isotopes, a single or a configuration-mixing IBM framework [59,60]. A simple IBM-1 Hamiltonian was applied for $\mathrm{Pt}$ nuclei in Ref. [59], leading to good agreement with the experimental data without a need for an intruder configuration. Moreover, in Ref. [60], both single (within IBM-1 framework) and configuration-mixing models were shown to give almost equivalent results for $\mathrm{Pt}$ isotopes being consistent with the experimental data, as long as the excitation energy is rather low (up to $E_{x} \sim 1.5 \mathrm{MeV}$ ). In this calculation, similarly to those in Refs. [59,60], the agreement between theoretical and experimental spectra, shown in Fig. 3, is reasonably good without introducing an intruder configuration. Once the intruder configuration is taken into account in the present framework, the number of free parameters to be fixed would largely increase. On the other hand, it is rather hard to identify a clear shape coexistence for the considered Pt nuclei in a microscopic level, as an HFB-PES in Fig. 1, indeed, does not exhibit any isolated local minimum other than the global one. Nevertheless, to what extent our result is changed by the configuration mixing, as well as a comparison between a single and a configuration-mixing calculation, is an interesting future issue that will be investigated further.

\section{B. Systematics of $B(E 2)$ ratios}

Once the boson wave functions corresponding to the excited states of a given nucleus are obtained, we are able to compute electromagnetic transitions, among which the reduced $E 2$ transition probabilities $B(E 2)$ are of particular importance. The $B(E 2)$ transition probabilities are given by [1]

$$
B\left(E 2 ; J \rightarrow J^{\prime}\right)=\frac{1}{2 J+1}\left|\left\langle J^{\prime} \| \hat{T}^{(E 2)}|| J\right\rangle\right|^{2},
$$

where $J$ and $J^{\prime}$ are the angular momenta for the initial and final states, respectively. The $E 2$ transition operator $\hat{T}^{(E 2)}$ is given by $\hat{T}^{(E 2)}=e_{\pi} \hat{Q}_{\pi}+e_{\nu} \hat{Q}_{\nu}$, with $e_{\pi}$ and $e_{\nu}$ being the boson effective charges. For simplicity, $e_{\pi}=e_{\nu}$ is assumed.

Here, the intrinsic quadrupole moment in the boson systems $Q_{I}$ is given as $[38,40]$

$$
Q_{I}=\frac{q\left[2\left(n_{\pi}+n_{\nu}\right) \beta_{B}-\sqrt{\frac{2}{7}}\left(n_{\pi} \chi_{\pi}+n_{\nu} \chi_{\nu}\right) \beta_{B}^{2}\right]}{1+\beta_{B}^{2}},
$$

where $q$ is an overall scaling factor. Since $Q_{I}$ is compared with $Q_{0}$ in Eqs. (5), and (12) leads one to $\beta_{B} \propto \beta$ when $\beta_{B}^{2}$ terms are neglected. Thus, the coefficient $C_{\beta}$ is related to the factor $q$. However, the factor $q$ does not mean the boson effective charge as $q$ is defined in the intrinsic state. In principle, the effective charges represent the effects beyond the mean field, such as core polarization, and should be determined independently of the underlying mean-field calculations. Therefore, in the current framework, we focus our discussion on the ratios of $B(E 2)$ values rather than on the quantities such as the absolute $B(E 2)$ values and the (spectroscopic) quadrupole moments.

The following $B(E 2)$ ratios are studied:

$$
\begin{aligned}
& R_{1}=B\left(E 2 ; 4_{1}^{+} \rightarrow 2_{1}^{+}\right) / B\left(E 2 ; 2_{1}^{+} \rightarrow 0_{1}^{+}\right), \\
& R_{2}=B\left(E 2 ; 2_{2}^{+} \rightarrow 2_{1}^{+}\right) / B\left(E 2 ; 2_{1}^{+} \rightarrow 0_{1}^{+}\right), \\
& R_{3}=B\left(E 2 ; 0_{2}^{+} \rightarrow 2_{1}^{+}\right) / B\left(E 2 ; 2_{1}^{+} \rightarrow 0_{1}^{+}\right), \\
& R_{4}=B\left(E 2 ; 2_{2}^{+} \rightarrow 0_{1}^{+}\right) / B\left(E 2 ; 2_{2}^{+} \rightarrow 2_{1}^{+}\right),
\end{aligned}
$$



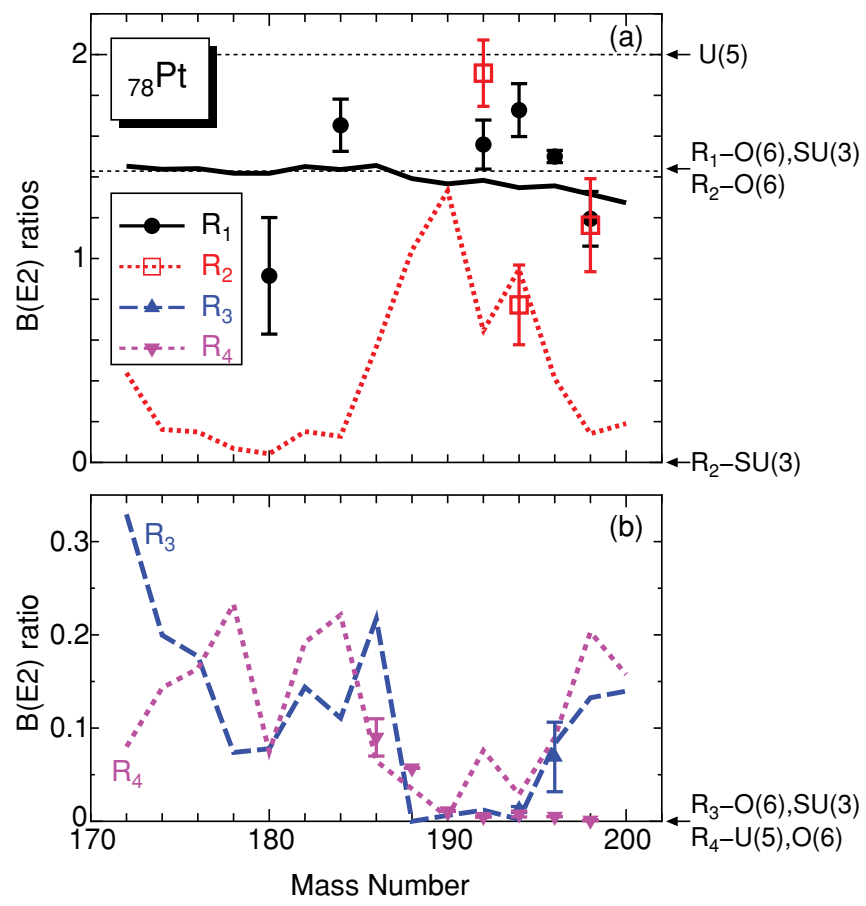

FIG. 4. (Color online) Reduced $E 2$ transition probabilities $B(E 2)$ of ${ }^{172-200} \mathrm{Pt}$ nuclei as functions of the mass number $A$. $B(E 2)$ ratios for relevant low-lying states (a) $R_{1}, R_{2}$ and (b) $R_{3}$ and $R_{4}$, defined in Eq. (13). Experimental data are taken from Refs. [49,69]. For more details, see the main text.

which are shown in Fig. 4 as functions of the mass number $A$. Note that the values of each dynamical symmetry limit [29], to be shown in the following, are those with infinite boson number.

The ratio $R_{1}$ in Fig. 4(a) is nearly constant all the way, being much below the $\mathrm{U}(5)$ limit of $\operatorname{IBM}\left(R_{1}=2\right)$, and is rather close to $R_{1}=10 / 7$, which is the $\mathrm{O}(6)$ and $\mathrm{SU}(3)$ limit of IBM. Thus, $R_{1}$ is not a sensitive observable to distinguish between axially symmetric and $\gamma$-soft nuclei. This is reasonable because the structural evolution between axially symmetric deformed and the $\gamma$-unstable shapes is shown to take place quite smoothly from the systematics of the mapped PESs (in Sec. III) and the derived IBM parameters (in Sec. IV). The flat behavior of the $R_{1}$ value for Pt isotopes in Fig. 4(a) differs from the one found, e.g., in Sm isotopes [74]. There, a sharp decrease of $R_{1}$ value can be seen in the line of U(5)-SU(3) shape/phase transition.

One can see also in Fig. 4(a) that, in contrast to the flat systematics of the $R_{1}$ value with respect to the mass number $A$, the ratio $R_{2}$ changes significantly and is relatively large for ${ }^{186-196} \mathrm{Pt}$ nuclei, being close to $\frac{10}{7}[\mathrm{O}(6)$ limit]. This is consistent with the softness of the PESs for these nuclei. Therefore, the quantity $R_{2}$ is quite sensitive to the shape evolution encountered in the PESs and can be thus considered as the best signature for $\gamma$ softness among $R_{1}-R_{4}$. There are not much available data overall, but the experimental $R_{2}$ value is also relatively large around ${ }^{192} \mathrm{Pt}$. For the nuclei ${ }^{176-184} \mathrm{Pt}$, the theoretical $R_{2}$ value is close to zero [the $\mathrm{SU}(3)$ limit] and slightly goes up from $A=174$ to 172 , probably approaching the $\mathrm{U}(5)$ vibrational limit $\left(R_{2}=2\right)$ in the vicinity of the neutron shell closure $N=82$.

Unlike the $R_{2}$ case, the calculated ratio $R_{3}$, shown in Fig. 4(b), does not change much with mass number $A$ and is close to zero $\left[\mathrm{O}(6)\right.$ and $\mathrm{SU}(3)$ limits of $\left.R_{3}\right]$ for ${ }^{188-196} \mathrm{Pt}$. From $A=180$, the $R_{3}$ turns to increase as we move toward the neutron shell closure $N=82$ and is expected to approach the U(5) limit $\left(R_{3}=2\right)$. The calculated $R_{3}$ value is, however, still much smaller than the experimental value at $A=198$. In fact, both the HFB and the mapped PESs for the nucleus ${ }^{198} \mathrm{Pt}$ display a weakly deformed shape, which somewhat differs from the vibrational feature expected from the corresponding experimental levels. The present $R_{3}$ value does not exhibit a drastic change observed in shape transitions in $A \sim 130 \mathrm{Ba}-\mathrm{Xe}$ and $A \sim 100 \mathrm{Ru}-\mathrm{Pd}$ isotopes, where the $E 2$ transition from the $0_{2}^{+}$state to the $2_{1}^{+}$is much enhanced [40].

Finally, the branching ratio $R_{4}$ in Fig. 4(b) also corresponds to a gradual shape transition. The present calculations suggest that the $R_{4}$ value is nearly zero [O $(6)$ limit] in the region where the nuclei are soft and where the $R_{2}$ ratio takes large values. The calculated $R_{4}$ ratio follows the experimental trend exhibiting increase from $A=190$ to 186, and becomes relatively larger for $A \leqslant 184$, where the PESs show stronger prolate deformation. Consistently with the evolution of the IBM PESs, the calculated $R_{4}$ values turn to approach the U(5) limit, which is also zero, for $A \leqslant 178$. Similarly to the $R_{3}$ case, a deviation from the vibrational character of the experimental data is found at $A=198$.

It should be emphasized that all the results for $B(E 2)$ values shown so far are quite consistent with the topologies of the PESs and with the derived IBM parameter values.

\section{Level schemes of selected nuclei}

As already mentioned in Sec. I, one of the main goals of this paper is to test the spectroscopic quality of the mapping procedure and the underlying (universal) Gogny-D1S EDF $[28,45]$, which have been described in Sec. II. Keeping this in mind, we will now turn our attention to a more detailed comparison between our results and the available experimental data for excitation spectra and $B(E 2)$ values. To this end, we select the nuclei ${ }^{184-194} \mathrm{Pt}$ as a representative sample corresponding to the mapped PESs shown in Fig. 1. The level schemes obtained for the nuclei ${ }^{184-194} \mathrm{Pt}$ are compared in Fig. 5 with available experimental data. The theoretical $B(E 2)$ values are shown also in Fig. 5 . Note that the $B\left(E 2 ; 2_{1}^{+} \rightarrow 0_{1}^{+}\right)$ value is normalized to the experimental value. The virtue of the present calculation is to give predicted $B(E 2)$ values for those nuclei that do not have enough $E 2$ information available. This is particularly useful in the cases of ${ }^{184} \mathrm{Pt},{ }^{186} \mathrm{Pt}$, and ${ }^{188} \mathrm{Pt}$, where the calculated spectra agree well with the experiment.

For clarity, we divide the explanation of the results shown in Fig. 5 into the following three categories, according to the tendencies of what we found in the PESs and the IBM parameters and the experimental data. The first is the prolatedeformed regime represented by the nuclei ${ }^{184,186} \mathrm{Pt}$, which exhibit a rotational character. Next, we will consider the isotopes ${ }^{188,190} \mathrm{Pt}$, which are apparently close to the critical 


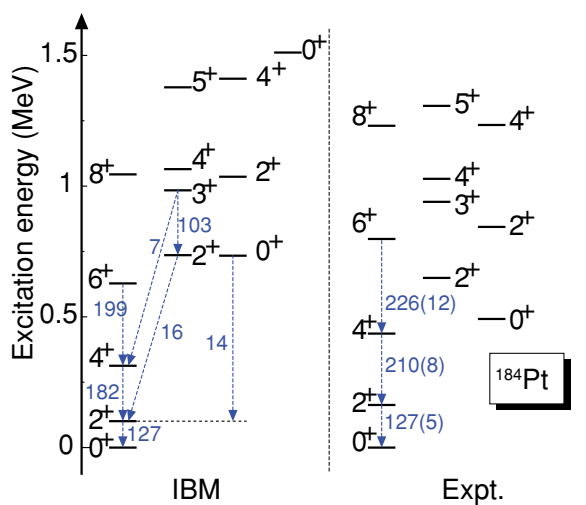

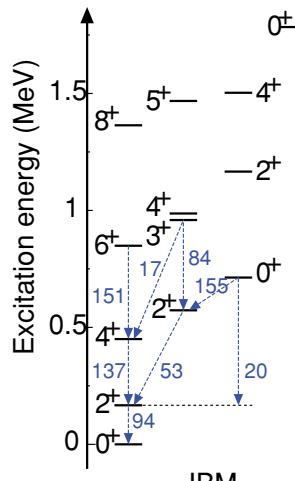

IBM

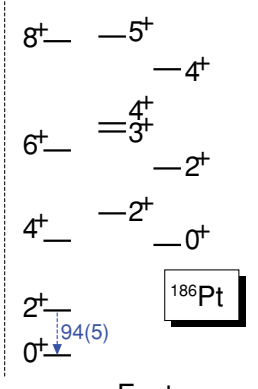

Expt.

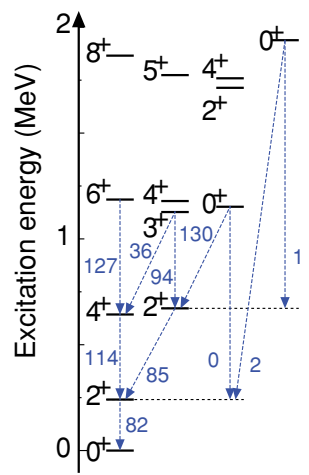

IBM

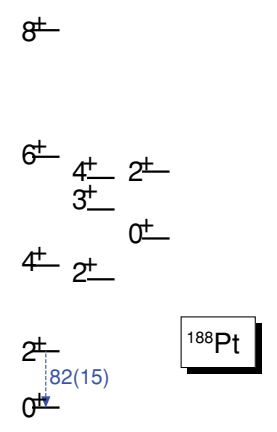

Expt.

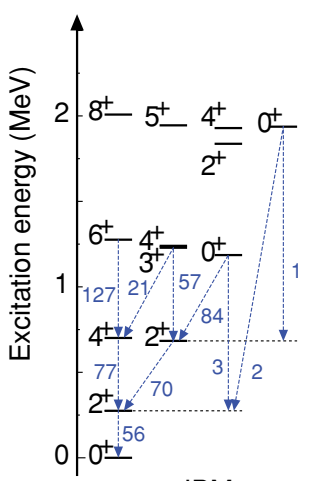

IBM
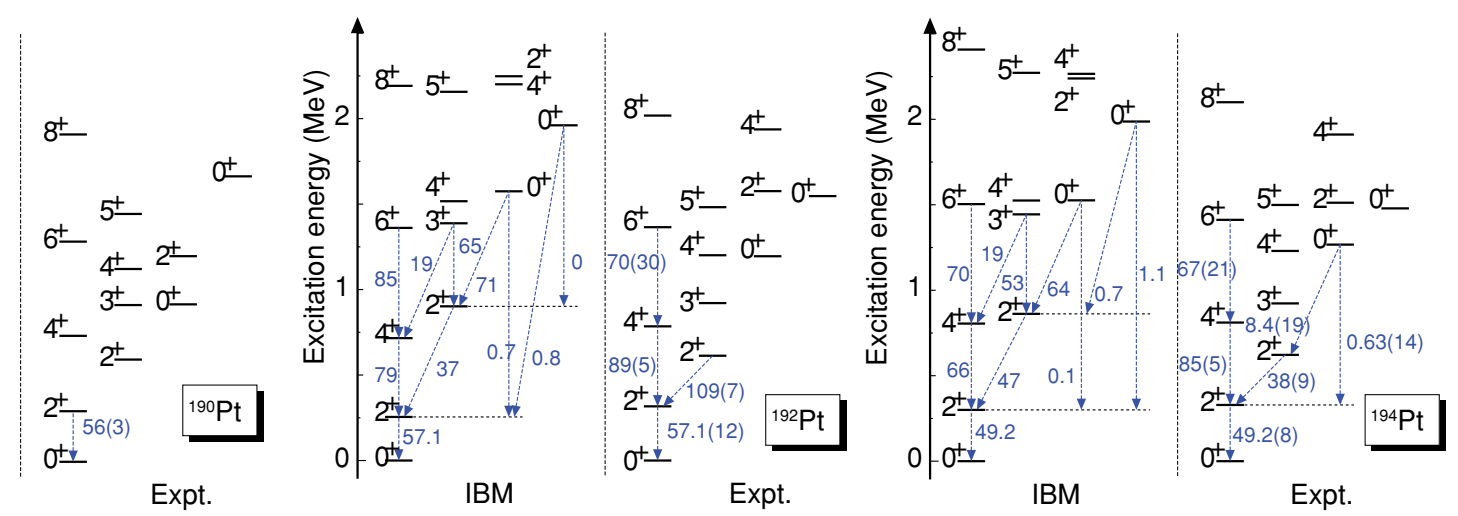

FIG. 5. (Color online) Level schemes for ${ }^{184-194} \mathrm{Pt}$ nuclei. The Gogny-D1S interaction is used. The theoretical $B(E 2)$ values (in Weisskopf units) for each nucleus are normalized to the experimental $B\left(E 2 ; 2_{1}^{+} \rightarrow 0_{1}^{+}\right)$value. Note that, in ${ }^{190} \mathrm{Pt}$, the theoretical $3_{1}^{+}$level is almost identical to, but is lower by only $10 \mathrm{keV}$ in energy than, the $4_{2}^{+}$level, where the $E 2$ transitions shown are those from the $3_{1}^{+}$state, not from the $4_{2}^{+}$state. For details, see the main text.

point of the prolate-to-oblate transition observed in the mapped PESs (see Fig. 1). Lastly, calculated and experimental results are compared for the nuclei ${ }^{192,194} \mathrm{Pt}$, which belong to the weakly oblate deformed regime. Note that, in Fig. 5, the energy scale is not common for all nuclei.

For ${ }^{184,186} \mathrm{Pt}$, the present calculation reproduces an overall pattern of the experimental spectra in all of the ground-state, quasi $-\beta$, and quasi $-\gamma$ bands fairly well. Interestingly enough, the band head energies, particularly the quasi- $\beta$ band head $0_{2}^{+}$, are much higher than the $4_{1}^{+}$level, compared to the experimental data. This indicates that, reflecting the topologies of the PESs in Fig. 1, the ${ }^{184,186} \mathrm{Pt}$ nuclei deviate from the $\gamma$-soft $\mathrm{O}(6)$ character and exhibit rather rotational features. The $0_{3}^{+}$ energies are predicted to be above the $4_{3}^{+}$energies in both nuclei.

On the other hand, both ${ }^{188} \mathrm{Pt}$ and ${ }^{190} \mathrm{Pt}$, with PESs that are quite flat along the $\gamma$ direction in Fig. 1, appear to be closer to the $\gamma$-unstable $\mathrm{O}(6)$ limit of the IBM than the two nuclei already mentioned above. The $2_{2}^{+}$and $4_{1}^{+}$levels lie close to each other in this study and, in the spirit of group theory, are supposed to have the same $\tau=2$ quantum number of the $\mathrm{O}(6)$ dynamical symmetry [29]. Similarly, in our calculations, the $6_{1}^{+}, 4_{2}^{+}, 3_{1}^{+}$, and $0_{2}^{+}$levels are almost degenerate and can be then grouped into the $\tau=3$ multiple. Along these lines, we can observe characteristic $E 2$ decay patterns that are quite consistent with the $\Delta \tau= \pm 1$ selection rule of the $\mathrm{O}(6)$ limit [29]. For instance, the transition from the $0_{2}^{+}$level (supposed to have $\tau=3$ ) to the $2_{2}^{+}$level (supposed to have $\tau=2$ ) is dominant over the one to $2_{1}^{+}$(supposed to have $\tau=1)$ in both ${ }^{188} \mathrm{Pt}$ and ${ }^{190} \mathrm{Pt}$. The trend characteristic of $\mathrm{O}(6)$ symmetry is clearly seen, particularly in ${ }^{190} \mathrm{Pt}$, where the sum of the parameters $\chi_{\pi}$ and $\chi_{v}$ almost vanishes as seen from Fig. 2(c). This means that the nucleus is close to the pure $\mathrm{O}(6)$ limit, and is consistent with the mapped PES in Fig. 1 that is nearly flat along the $\gamma$ direction. Nevertheless, the structure of the corresponding experimental $\gamma$ band appears to have a more triaxial nature, where the $3_{1}^{+}$and the $4_{2}^{+}$levels are apart from each other. As we have anticipated in Sec. III, this deviation arises partly due to the difference of the position of energy minimum between Gogny-D1S PESs and the corresponding IBM PESs of Fig. 1.

For ${ }^{192,194} \mathrm{Pt}$ nuclei, the theoretical $\gamma$-band structure still looks like that of $\mathrm{O}(6)$ symmetry. What is of particular interest here is that, for both ${ }^{192,194} \mathrm{Pt}$, the relative location of the quasi$\beta$ band head $0_{2}^{+}$energy is reproduced fairly well lying close to the $4_{2}^{+}$level. In addition, for ${ }^{194} \mathrm{Pt}$, the present calculation suggests that the $0_{2}^{+} \rightarrow 2_{2}^{+} E 2$ transition is dominant over the $\mathrm{O}_{2}^{+} \rightarrow 2_{1}^{+} E 2$ transition, which, although there is quantitative deviation, agrees with the experimental trend. The reason why such a quantitative difference occurs may be discussed in the future. Compared to the experimental data, the theoretical quasi $-\gamma$ band is rather stretched and the band head $2_{2}^{+}$energy is somewhat large. The calculated $0_{2}^{+}$energy is also higher than the experimental energy, in particular for ${ }^{192} \mathrm{Pt}$. Accordingly, 
the theoretical $B\left(E 2 ; 2_{2}^{+} \rightarrow 2_{1}^{+}\right)$value is much smaller than the experimental value with respect to the $B\left(E 2 ; 2_{1}^{+} \rightarrow 0_{1}^{+}\right)$ value. The deviations occur due to the derived $\kappa$ value, which is somewhat larger than the phenomenological value [49]. For relatively high-lying sideband $2_{3}^{+}$and $4_{3}^{+}$energies, the calculated results may not seem to be very reliable, because even the ordering of these levels is not reproduced for ${ }^{192} \mathrm{Pt}$.

\section{SUMMARY}

To summarize, spectroscopic calculations have been carried out for the Pt isotopic chain in terms of the interacting boson model Hamiltonian derived microscopically based on the (constrained) Hartree-Fock-Bogoliubov approach with the Gogny-D1S energy density functional.

The Gogny-HFB calculations provide the potential energy surface, which reflects, to a good extent, many-nucleon dynamics of surface deformation with quadrupole degrees of freedom and structural evolution in a given isotopic chain. By following the procedure proposed in Ref. [38], the PES of the Gogny-D1S EDF is mapped onto the corresponding bosonic PES, and can be then utilized as a guideline for determining the parameters of the IBM Hamiltonian. This enables one to calculate the spectroscopic observables with good quantum numbers (i.e., the angular momentum and the particle number) in the laboratory system without adjustment of levels.

By this approach, global tendencies of the experimental low-lying spectra of ${ }^{172-200} \mathrm{Pt}$ nuclei are reproduced quite well not only for the ground state, but also for sidebands of mainly open-shell nuclei. It has been shown that shape/phase transition occurs quite smoothly from prolate-to-oblate deformations as a function of $N$ in the considered nuclei ${ }^{186-192} \mathrm{Pt}$, where the $\gamma$ instability plays an essential role. From the analysis in Fig. 1, the change of the mapped IBM PESs in the $\gamma$ direction has been more vividly seen than in the $\beta$ direction, similar to the corresponding Gogny-HFB PESs. This is consistent with the conclusions in our earlier work [42] and also with many others along the same line. We have shown that the calculated spectra and the $B(E 2)$ ratios behave consistently with the evolution of the topologies of the mapped PES's and with the systematics of the derived IBM parameters as functions of the neutron number $N$. These derived parameters are qualitatively quite similar to the existing phenomenological IBM studies [47,49].
By studying the level schemes in detail in comparison with the available experimental data, our calculation agrees with the data fairly nicely and reflects the algebraic aspects of the IBM, e.g., the $\Delta \tau= \pm 1$ selection rule of the $E 2$ decay patterns. We have also made predictions on some $E 2$ transition patterns. These behaviors of the $B(E 2)$ may need to be examined experimentally, particularly for lighter $A \lesssim 190$ nuclei, for which there is currently not much data available.

The evolution of the ground-state shape as a function of both $N$ and $Z$ has been studied within neighboring isotopic chains such as Os, W, Hf, and $\mathrm{Yb}$ [28]. More systematic analysis is in order for these nuclei by more extensive application of the present approach. It should then be of interest to study how the corresponding spectra and transition probabilities behave.

On the other hand, the IBM Hamiltonian of Eq. (6) has a rather simple form consisting of single- $d$-boson operator and the quadrupole-quadrupole interaction between proton and neutron bosons. The results provided by the present Hamiltonian were shown to be already quite promising. However, more studies may be necessary in the future for further refinement, e.g., in describing the detailed structure of the quasi $-\gamma$ band. Work along this line is in progress. Inclusions of intruder configuration and cubic terms also will be of great interest.

\section{ACKNOWLEDGMENTS}

We thank D. Vretenar for valuable discussions. This work was supported in part by a Grant-in-Aid for Scientific Research (A) 20244022 and by a Grant-in-Aid from JSPS (No. 217368). K.N. is supported by a JSPS program. Part of this work was carried out during his visit to the European Center for Theoretical Nuclear Physics and Related Areas (ECT*). He acknowledges ECT* and A. Richter for their kind hospitality. The work of L.M.R and P.S was supported by MICINN (Spain) under research Grants No. FIS2008-01301, No. FPA2009-08958, and No. FIS2009-07277, as well as by Consolider-Ingenio 2010 Programs CPAN CSD2007-00042 and MULTIDARK CSD2009-00064. R.R. acknowledges the support received within the framework of the FIDIPRO program (Academy of Finland and University of Jyväskylä) and thanks J. Äysto and R. Julin, as well as the experimental teams of the University of Jyväskylä (Finland), for warm hospitality and encouraging discussions.
[1] A. Bohr and B. R. Mottelson, Nuclear Structure (Benjamin, New York, 1969 and 1975), Vols. I and II.

[2] A. Bohr, Mat. Fys. Medd. Dan. Vid. Selsk. 26, No. 14 (1952); A. Bohr and B. R. Mottelson, ibid. 27, No. 16 (1953).

[3] P. Ring and P. Schuck, The Nuclear Many-Body Problem (Springer, Berlin, 1980).

[4] P. Cejnar, J. Jolie, and R. F. Casten, Rev. Mod. Phys. 82, 2155 (2010).

[5] J. L. Wood, K. Heyde, W. Nazarewicz, M. Huyse, and P. Van Duppen, Phys. Rep. 215, 101 (1992).

[6] T. R. Werner, J. Dobaczewski, M. W. Guidry, W. Nazarewicz, and J. A. Sheikh, Nucl. Phys. A 578, 1 (1994).
[7] M. Bender, P.-H. Heenen, and P.-G. Reinhard, Rev. Mod. Phys. 75, 121 (2003).

[8] S. Cwiok, P.-H. Heenen, and W. Nazarewicz, Nature (London) 433, 705 (2005).

[9] L. M. Robledo, R. R. Rodríguez-Guzmán, and P. Sarriguren, Phys. Rev. C 78, 034314 (2008).

[10] P. Sarriguren, R. Rodríguez-Guzmán, and L. M. Robledo, Phys. Rev. C 77, 064322 (2008).

[11] R. Rodríguez-Guzmán and P. Sarriguren, Phys. Rev. C 76, 064303 (2007).

[12] J. L. Egido, L. M. Robledo, and R. R. Rodríguez-Guzmán, Phys. Rev. Lett. 93, 082502 (2004). 
[13] W. Nazarewicz, Nucl. Phys. A 574, 27c (1994).

[14] I. Hamamoto and B. R. Mottelson, Phys. Rev. C 79, 034317 (2009).

[15] R. Julin, K. Helariutta, and M. Muikku, J. Phys. G: Nucl. Part. Phys. 27, R109 (2001).

[16] G. D. Dracoulis, A. E. Stuchbery, A. P. Byrne, A. R. Poletti, S. J. Polotti, J. Gerl, and R. A. Bark, J. Phys. G 12, L97 (1986); G. D. Dracoulis et al., Phys. Rev. C 44, R1246 (1991).

[17] P. M. Davidson et al., Nucl. Phys. A 657, 219 (1999).

[18] C. Y. Wu et al., Nucl. Phys. A 607, 178 (1996).

[19] Zs. Podolyák et al., Phys. Lett. B 491, 225 (2000).

[20] M. Pfützner et al., Phys. Rev. C 65, 064604 (2002).

[21] M. Caamaño et al., Eur. Phys. J. A 23, 201 (2005).

[22] J. Dechargé, M. Girod, and D. Gogny, Phys. Lett. B 55, 361 (1975),

[23] J. Dechargé and D. Gogny, Phys. Rev. C 21, 1568 (1980).

[24] T. H. R. Skyrme, Nucl. Phys. 9, 615 (1959).

[25] D. Vautherin and D. M. Brink, Phys. Rev. C 5, 626 (1972).

[26] D. Vretenar, A. V. Afanasjev, G. A. Lalazissis, and P. Ring, Phys. Rep. 409, 101 (2005).

[27] J. L. Egido, J. Lessing, V. Martin, and L. M. Robledo, Nucl. Phys. A 594, 70 (1995).

[28] L. M. Robledo, R. Rodríguez-Guzmán, and P. Sarriguren, J. Phys. G: Nucl. Part. Phys. 36, 115104 (2009).

[29] A. Arima and F. Iachello, Phys. Rev. Lett. 35, 1069 (1975); F. Iachello and A. Arima, The Interacting Boson Model (Cambridge University Press, Cambridge, 1987).

[30] T. Otsuka, A. Arima, F. Iachello, and I. Talmi, Phys. Lett. B 76, 139 (1978); T. Otsuka, A. Arima, and F. Iachello, Nucl. Phys. A 309, 1 (1978).

[31] T. Otsuka, in Algebraic Approaches to Nuclear Structure, edited by R. F. Casten (Harwood, Chur, Switzerland, 1993), p. 195.

[32] T. Mizusaki and T. Otsuka, Prog. Theor. Phys. Suppl. 125, 97 (1997).

[33] M. Deleze, S. Drissi, J. Kern, P. A. Tercier, J. P. Vorlet, J. Rikovska, T. Otsuka, S. Judge, and A. Williams, Nucl. Phys. A 551, 269 (1993).

[34] K. Allaart, G. Bonsignori, M. Savoia, and V. Paar, Nucl. Phys. A 458, 412 (1986).

[35] Y. K. Gambhir, P. Ring, and P. Schuck, Phys. Rev. C 25, 2858 (1982).

[36] T. Otsuka, Phys. Lett. B 138, 1 (1984).

[37] T. Otsuka and N. Yoshinaga, Phys. Lett. B 168, 1 (1986).

[38] K. Nomura, N. Shimizu, and T. Otsuka, Phys. Rev. Lett. 101, 142501 (2008).

[39] P. Bonche, H. Flocard, and P.-H. Heenen, Comput. Phys. Commun. 171, 49 (2005).

[40] K. Nomura, N. Shimizu, and T. Otsuka, Phys. Rev. C 81, 044307 (2010).

[41] J. F. Berger, M. Girod, and D. Gogny, Nucl. Phys. A 428, 23c (1984).

[42] R. Rodriguez-Guzman, P. Sarriguren, L. M. Robledo, and J. E. Garcia-Ramos, Phys. Rev. C 81, 024310 (2010).

[43] F. Chappert, M. Girod, and S. Hilaire, Phys. Lett. B 668, 420 (2008).

[44] S. Goriely, S. Hilaire, M. Girod, and S. Peru, Phys. Rev. Lett. 102, 242501 (2009).
[45] J.-P. Delaroche, M. Girod, J. Libert, H. Goutte, S. Hilaire, S. Peru, N. Pillet, and G. F. Bertsch, Phys. Rev. C 81, 014303 (2010).

[46] J. A. Cizewski, R. F. Casten, G. J. Smith, M. L. Stelts, W. R. Kane, H. G. Borner, and W. F. Davidson, Phys. Rev. Lett. 40, 167 (1978).

[47] R. F. Casten and J. A. Cizewski, Nucl. Phys. A 309, 477 (1978).

[48] P. D. Duval and B. R. Barrett, Phys. Rev. C 23, 492 (1981).

[49] R. Bijker, A. E. L. Dieperink, O. Scholten, and R. Spanhoff, Nucl. Phys. A 344, 207 (1980).

[50] Zs. Podolyák et al., Phys. Rev. C 79, 031305(R) (2009).

[51] K. Kummer and M. Baranger, Nucl. Phys. A 122, 273 (1968).

[52] J.-P. Delaroche et al., Phys. Rev. C 50, 2332 (1994).

[53] T. Nikšić, P. Ring, D. Vretenar, Y. Tian, and Zhong-yu Ma, Phys. Rev. C 81, 054318 (2010).

[54] A. N. Andreyev et al., Nature (London) 405, 430 (2005).

[55] T. Duguet, M. Bender, P. Bonche, and P.-H. Heenen, Phys. Lett. B 559, 201 (2003).

[56] M. Bender, P. Bonche, T. Duguet, and P.-H. Heenen, Phys. Rev. C 69, 064303 (2004).

[57] R. R. Rodriguez-Guzman, J. L. Egido, and L. M. Robledo, Phys. Rev. C 69, 054319 (2004).

[58] P. D. Duval and B. R. Barrett, Nucl. Phys. A 376, 213 (1983).

[59] E. A. McCutchan, R. F. Casten, and N. V. Zamfir, Phys. Rev. C 71, 061301(R) (2005).

[60] J. E. Garcia-Ramos and K. Heyde, Nucl. Phys. A 825, 39 (2009).

[61] A. E. L. Dieperink and O. Scholten, Nucl. Phys. A 346, 125 (1980).

[62] J. N. Ginocchio and M. Kirson, Nucl. Phys. A 350, 31 (1980).

[63] A. Bohr and B. R. Mottelson, Phys. Scr. 22, 468 (1980).

[64] G. Kaiser, A Friendly Guide to Wavelets (Birkhäser, Boston, 1994).

[65] P. Bonche, J. Dobaczewski, H. Flocard, P.-H. Heenen, and J. Meyer, Nucl. Phys. A 510, 466 (1990).

[66] Z. P. Li, T. Nikšić, D. Vretenar, and J. Meng, Phys. Rev. C 81, 034316 (2010); Z. P. Li, T. Nikšić, D. Vretenar, J. Meng, G. A. Lalazissis, and P. Ring, ibid. 79, 054301 (2009).

[67] K. Nomura, T. Otsuka, N. Shimizu, and L. Guo, arXiv:1011.1056.

[68] Brookhaven National Nuclear Data Center (NNDC) [http://www.nndc.bnl.gov/].

[69] C. M. Baglin, Nucl. Data Sheets 96, 611 (2002); S. Balraj, ibid. 75, 199 (1995); E. Browne and H. Junde, ibid. 87, 15 (1999); M. S. Basunia, ibid. 107, 791 (2006); E. Achterberg et al., ibid. 110, 1473 (2009); S.-C. Wu and H. Niu, ibid. 100, 483 (2003); B. Singh and R. B. Firestone, ibid. 74, 383 (1995); C. M. Baglin, ibid. 111, 275 (2010); C. M. Baglin, ibid. 99, 1 (2003); B. Singh, ibid. 95, 387 (2002); B. Singh, ibid. 99, 275 (2003); C. M. Baglin, ibid. 84, 717 (1998); B. Singh, ibid. 107, 1531 (2006); H. Xiaolong, ibid. 108, 1093 (2007); 110, 2533 (2007); F. G. Kondeva and S. Lalkovski, ibid. 108, 1471 (2007).

[70] T. Otsuka and N. Yoshida, JAERI-M Report No. 85 (unpublished).

[71] A. S. Davydov and G. F. Filippov, Nucl. Phys. 8, 237 (1958).

[72] K. Heyde, P. Van Isacker, M. Waroquier, and J. Moreau, Phys. Rev. C 29, 1420 (1984).

[73] R. F. Casten, P. von Brentano, K. Heyde, P. Van Isacker, and J. Jolie, Nucl. Phys. A 439, 289 (1985).

[74] O. Scholten, F. Iachello, and A. Arima, Ann. Phys. (NY) 115, 325 (1978). 